\title{
The first observed stellar X-ray flare oscillation: Constraints on the flare loop length and the magnetic field
}

\author{
U. Mitra-Kraev, L. K. Harra, D. R. Williams, and E. Kraev \\ Mullard Space Science Laboratory, University College London, Holmbury St. Mary, Dorking, Surrey RH5 6NT, UK \\ e-mail: umk@mssl.ucl.ac.uk \\ Received 7 February 2005 / Accepted 12 March 2005

\begin{abstract}
We present the first X-ray observation of an oscillation during a stellar flare. The flare occurred on the active M-type dwarf AT Mic and was observed with XMM-Newton. The soft X-ray light curve $(0.2-12 \mathrm{keV})$ is investigated with wavelet analysis. The flare's extended, flat peak shows clear evidence for a damped oscillation with a period of around $750 \mathrm{~s}$, an exponential damping time of around $2000 \mathrm{~s}$, and an initial, relative peak-to-peak amplitude of around 15\%. We suggest that the oscillation is a standing magneto-acoustic wave tied to the flare loop, and find that the most likely interpretation is a longitudinal, slow-mode wave, with a resulting loop length of $(2.5 \pm 0.2) \times 10^{10} \mathrm{~cm}$. The local magnetic field strength is found to be $105 \pm 50 \mathrm{G}$. These values are consistent with (oscillation-independent) flare cooling time models and pressure balance scaling laws. Such a flare oscillation provides an excellent opportunity to obtain coronal properties like the size of a flare loop
\end{abstract} \\ or the local magnetic field strength for the otherwise spatially-unresolved star.
}

Key words. stars: coronae - stars: flare - stars: magnetic fields - stars: oscillations - X-rays: stars - stars: individual: AT Mic

\section{Introduction}

Oscillations in the solar corona have by now been observed for many years. Wavelength regimes ranging from radio to hard X-rays have been investigated in the search for evidence of waves. Table 1 in Aschwanden et al. (1999) provides a summary and description of the different periods found $(0.02$ to $1000 \mathrm{~s}$ ). Most of these waves have been explained by magneto-hydrodynamic (MHD) oscillations in coronal loops, see Roberts (2000) for a detailed review of waves and oscillations in the corona.

One of the most exciting aspects of "coronal seismology" is that it potentially provides us with the capability for determining the magnetic field strength in the corona (Roberts et al. 1984), as well as, in the stellar case, with information on otherwise unresolved spatial scales, e.g., flare loop lengths. It is notoriously difficult to measure the magnetic field strength in the corona because of the very high speeds of coronal electrons, which broaden spectral lines far beyond the width of Zeeman splitting. Techniques using both near-infrared emission lines and radio observations are successful, but have poor spatial resolution. Indirect methods are commonly used, such as the extrapolations of the coronal magnetic field from the photospheric magnetic field, which in turn can be measured using the Zeeman effect.

Many of the observations of waves have been determined from variations in intensity. However, a huge step forward was achieved in solar coronal physics due to the high spatial resolution available with the Transition Region and Coronal Explorer
(TRACE, Handy et al. 1999). Aschwanden et al. (1999) observed the first spatial displacement oscillations of coronal loops. It was suggested that these oscillations were triggered by a fast-mode shock from a flare site, and they were interpreted as standing fast kink-mode waves.

Nakariakov \& Ofman (2001) made use of such flare-related spatial oscillations to determine the magnetic field strength $(13 \pm 9 \mathrm{G})$, which in the case of a standing kink wave is related to the period of the oscillation, the density of the loop, and the length of the loop. Another model used to derive the magnetic field strength from loop oscillations was put forward by Zaĭtsev \& Stepanov (1989), which assumes that the oscillation is triggered by a centrifugal force, generated by the evaporating chromospheric plasma moving upward along the magnetic field. In this case, the magnetic field strength is given by the amplitude of the oscillation, as well as the loop density and temperature. The model also predicts loop lengths if collisional damping is assumed. (Or vice versa, density and temperature, if the loop length is known.)

Although waves are observed across the electromagnetic spectrum on the Sun, observations on other stars are rarer. The principal reason for this is that the Sun can be spatially resolved, whereas on stars, the signal for the wave must be strong enough to be observed above the full disk emission. The first oscillation associated with a stellar flare was reported by Rodonò (1974), who observed the flare star II Tau with highspeed optical photometry, and found a long-lived oscillation during flare decay with a mean period of $13 \mathrm{~s}$. In a series of 
papers in the late 1980's and early 1990's, Andrews and coworkers (e.g., Andrews 1990) presented optical observations of dMe flare stars, where they observed quasi-periodicities with periods of a few tens of seconds, and put forward the idea that these were coronal loop oscillations. Mullan et al. (1992) also found optical oscillations (periods of the order of a few minutes) in dMe stars, concluding that they more likely arise from a coronal than from a photospheric origin. In a later paper, Mullan \& Johnson (1995) found oscillations in X-ray data of dMe stars. These oscillations have periods in the range of several tens to a few hundreds of seconds. They were not associated with any flare, though they were interpreted as coronal loop oscillations. Optical stellar flare oscillations have been observed by Mathioudakis et al. (2003), who found a period of $220 \mathrm{~s}$ in the decay phase of a white-light flare on the RS CVn binary II Peg.

In this work, we investigate the first X-ray observation of an oscillation during a stellar flare. Section 2 describes the target, AT Mic, as well as the observation. In Sect. 3, we present the data analysis, determining the period and amplitude of the oscillation through wavelet analysis. In Sect. 4, we determine the magnetic field strength and the length of the coronal loop by assuming that the oscillation is due to a magneto-acoustic wave. As a validity check, the value of loop length was compared to the value determined from a radiative cooling model, as well as from pressure balance scaling laws. The latter also gives an independent estimate of the magnetic field strength. Finally, we discuss the results and give conclusions (Sect. 5).

\section{Target and observation}

\subsection{Target}

AT Mic (GJ 799A/B) is an M-type binary dwarf, with both stars of the same spectral type (dM4.5e+dM4.5e). Both components of the binary flare frequently. The radius of AT Mic given by Lim et al. (1987) is $2.6 \times 10^{10} \mathrm{~cm}$, using a stellar distance of 8.14 pc (Gliese \& Jahreiss 1991). Correcting for the newer value for the distance from HIPPARCOS $(10.2 \pm 0.5 \mathrm{pc}$, Perryman et al. 1997), and using the fact that the stellar radius is proportional to the stellar distance for a given luminosity and spectral class, we obtain a stellar radius of $r_{\star}=3.3 \times 10^{10} \mathrm{~cm}=$ $0.47 r_{\odot}$. The mass of AT Mic given by Lim et al. (1987) is $m_{\star}=0.4 m_{\odot}$.

\subsection{Observation}

For our analysis we used the XMM-Newton (Jansen et al. 2001) observations of AT Mic on 16 October 2000 during revolution 156. Raassen et al. (2003) have analysed this data spectroscopically, obtaining elemental abundances, temperatures, densities and emission measures, while a comparative flare analysis between X-ray and simultaneously observed ultraviolet emissions can be found in Mitra-Kraev et al. (2005). Here, we solely used the $0.2-12 \mathrm{keV}$ X-ray data from the pn-European Photon Imaging Camera (EPIC-pn, Strüder et al. 2001).

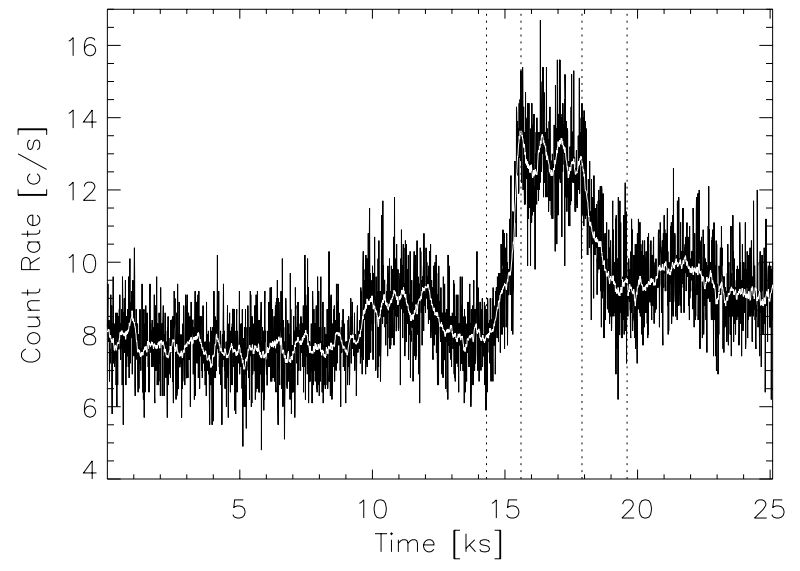

Fig. 1. The AT Mic 0.2-12 keV X-ray 10-s bin light curve (shown in black). Overplotted in white is the same light curve smoothed with a sliding time window of $200 \mathrm{~s}$. The time is in ks, starting from the beginning of the observation (2000-10-16 00:42:00). The vertical dotted lines mark the flare start, end of rise phase, end of extended top phase and end of the flare.

The observation started at 00:42:00 and lasted for $25.1 \mathrm{ks}$ $(\sim 7 \mathrm{~h})$. Figure 1 shows the AT Mic light curve. A large flare, starting $\sim 15 \mathrm{ks}$ into the observation, increases the count-rate from flare onset to flare peak by a factor of 1.7, and lasts for $1 \mathrm{~h} 25 \mathrm{~min}$. It shows a steep rise (rise time $\tau_{\mathrm{r}}=1300 \mathrm{~s}$ ) and decay (decay time $\tau_{\mathrm{d}}=1700 \mathrm{~s}$ ). There is an extended peak to this flare, which shows clear oscillatory behaviour. Applying a multi-temperature model, Raassen et al. (2003) obtain a best fit with a mean flare temperature $T=24 \pm 4 \mathrm{MK}$ and a quiescent temperature $T_{\mathrm{e}}=13 \pm 1 \mathrm{MK}$, and from the $\mathrm{O}$ VII line ratio a flare and quiescent electron density of $n=4_{-3}^{+5} \times 10^{10} \mathrm{~cm}^{-3}$ and $n_{\mathrm{e}}=(1.9 \pm 1.5) \times 10^{10} \mathrm{~cm}^{-3}$, respectively. The total flare and quiescent emission measures are $E M=(19.5 \pm 0.8) \times$ $10^{51} \mathrm{~cm}^{-3}$ and $E M_{\mathrm{e}}=(12.2 \pm 0.5) \times 10^{51} \mathrm{~cm}^{-3}$. The total emitted energy in the $0.2-12 \mathrm{keV}$ band of this flare is $\sim 6 \times$ $10^{32}$ erg (Mitra-Kraev et al. 2005).

\section{Analysis}

\subsection{Data preparation}

For the extraction of the EPIC-pn light curve we used the XMM-Newton Science Analysis System (SAS) version 6.0. Light curves with a cadence of $1 \mathrm{~s}$ were extracted for concentric regions around the source (20:41:51.156, -32:26:11.02, ICRS 2000 coordinates), and a background, off-set from the source on the same detector chip (20:41:48.428, -32:29:00.66); both regions used a radius of 41.325 arcsec. The selected energy range is $0.2-12 \mathrm{keV}(\lambda=1-62 \AA)$. The obtained errors for the count rates follow Poisson statistics for all data points. The background light curve is subtracted from the source light curve. Data missing due to telemetry absences are identified by obtaining the 1-s light curve of the entire detector chip; they manifest as time intervals with zero count rate. At such times, the background-subtracted raw light curve is interpolated. This light curve is then re-binned to 10-s bins and used for further analysis. 


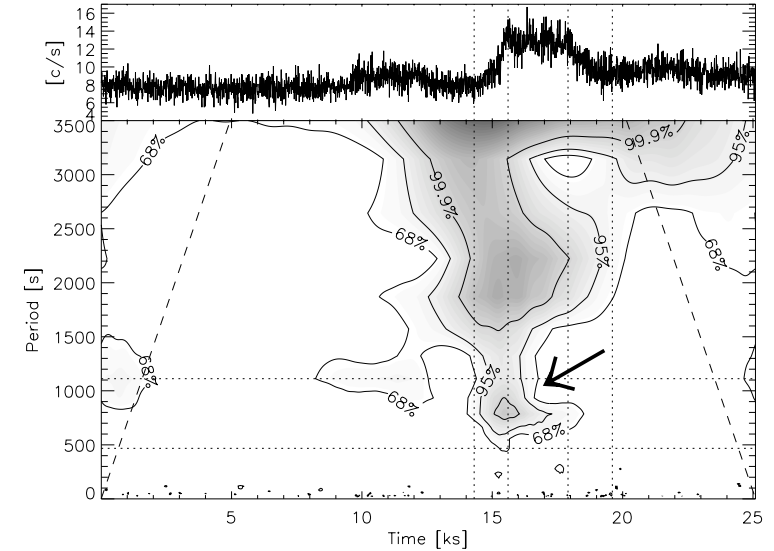

Fig. 2. The upper panel shows the 10-s bin light curve. The lower panel displays the high-frequency wavelet coefficients of the continuous wavelet transform of the above light curve, using a Morlet wavelet (see Sect. 3.2). The contours give the 68\%, 95\% and 99.9\% significance levels. The dashed lines represent the cone of influence. The vertical dotted lines are the same as in Fig. 1. The arrow points to the flare oscillation in the wavelet domain and the horizontal dotted lines mark the division between the high (noise), medium (oscillation) and low frequency ranges.

\subsection{Continuous wavelet transform and frequency band decomposition}

We apply a continuous wavelet transform (CWT) to the 10-s light curve, following the approach laid out by Torrence \& Compo (1998). For our analysis, we use the Morlet wavelet $\Psi_{0}(\eta)=\pi^{-1 / 4} \mathrm{e}^{i 6 \eta} \mathrm{e}^{-\eta^{2} / 2}$. Figure 2 displays the CWT. The vertical dotted lines denote the start (14.3 ks), the end of the rise phase $(15.6 \mathrm{ks})$, the beginning of the decay phase (17.9 ks) and the end (19.6 ks) of the flare. The contours give significance levels of $68 \%, 95 \%$ and $99.9 \%$, respectively. At flare rise, the power is enhanced at all timescales in the CWT. The bold arrow points to the flare oscillation, which causes a local maximum in the wavelet coefficient plane. It has a period between 500 and $1200 \mathrm{~s}$ and occurs during flare maximum, with a significance level of $>99.9 \%$. Note that there is another local maximum of $68 \%$ significance at a period of $\sim 1000 \mathrm{~s}$ occurring during a weaker flare at around $t=10 \mathrm{ks}$, which suggests that this earlier flare might oscillate as well. A third, minor flare at $t=22 \mathrm{ks}$, on the other hand, shows no oscillation. Here, we investigate only the major oscillation during the largest flare.

We split the CWT into three frequency bands with periods of $P>1200 \mathrm{~s}, 500 \mathrm{~s}<P<1200 \mathrm{~s}$, and $10 \mathrm{~s}<P<500 \mathrm{~s}$, so that the middle band encompasses only the oscillation range. The light curve is reconstructed for the three bands separately. Figure 3 shows these decomposed light curves (upper three panels), and their sum, the reconstructed light curve (lowest panel). As the decomposition is a linear transform, it is easy to derive the standard errors for the decomposed light curves (see Appendix A for the error treatment in CWT analysis), they are represented by the shaded areas around each light curve. Figure 3 demonstrates how the oscillation has been separated from the flare profile, allowing the oscillation to be analysed separately.

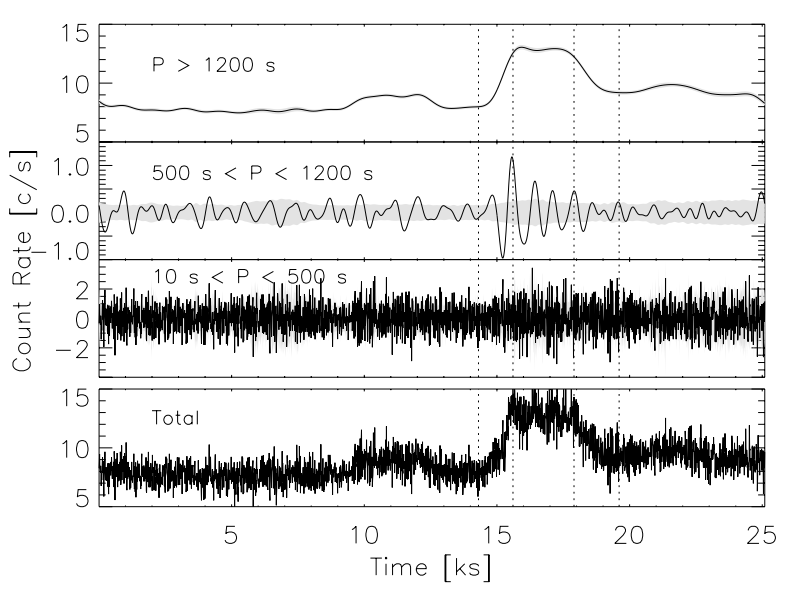

Fig. 3. The reconstructed light curve divided into three frequency bands, low $(P>1200 \mathrm{~s})$, medium $(500 \mathrm{~s}<P<1200 \mathrm{~s}$, including the oscillation) and high $(10 \mathrm{~s}<P<500 \mathrm{~s}$, mainly noise); together they add up to the original data (lowest panel). The shaded areas show the standard errors. The vertical dotted lines are the same as in Fig. 1. A coherent oscillation is easily identified during flare peak.

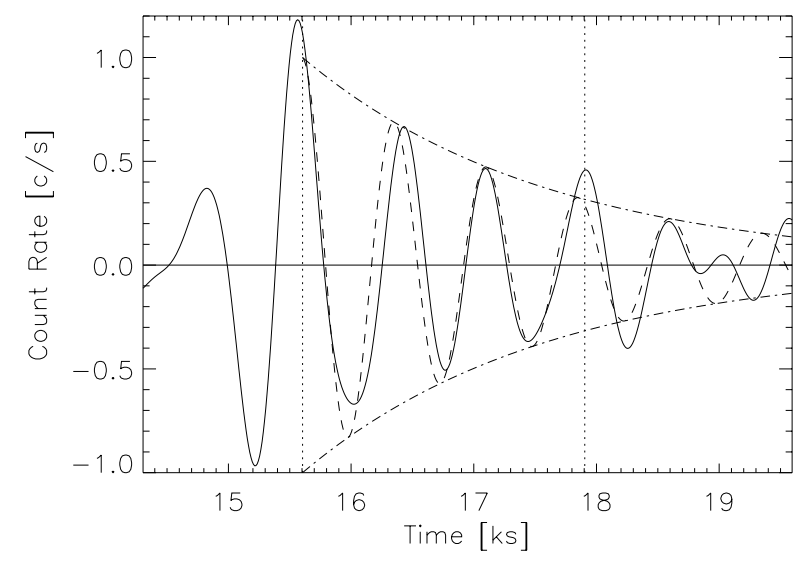

Fig. 4. The flare part of the $P=500 \ldots 1200 \mathrm{~s}$ reconstructed light curve (Fig. 3, second panel). The vertical dotted lines bound the flare top. The solid line shows the reconstructed light curve from the data, whereas the dashed line is a damped sine curve (bounded by the exponential envelope, dashed-dotted line) with an oscillation period of $P=750 \mathrm{~s}$ and an exponential decay time of $\tau=2000 \mathrm{~s}$. The relative peak-to-peak amplitude is initially $15 \%$.

Figure 4 shows the isolated oscillation (solid line) to which we fit a damped sine curve (dashed line). The fitted curve has an oscillation with a period of $P=750 \mathrm{~s}$, an exponential decay (damping) time of $\tau=2000 \mathrm{~s}$, and an amplitude of 1 count s$^{-1}$, which gives, with an average count rate of 13 counts $\mathrm{s}^{-1}$ during flare peak (from the low-frequency light curve), a peak-to-peak amplitude of $\Delta I / I=15 \%$.

A continuous wavelet transform is able to provide us with all our aims simultaneously: the wave is located in time and frequency ( $=1 /$ period), it is naturally isolated from the underlying flare profile and thus the amplitude and damping are easily available from the fit parameters for a damped sine wave. The low signal-to-noise ratio is dealt with by disentangling the high frequencies from the lower oscillating frequency. In particular, this provides us with a better estimate for the amplitude. While 
the period derived from the smoothed light curve is broadly the same as the period obtained from wavelet analysis, the amplitude $\Delta I / I=\left(I_{\max }-I_{\min }\right)\left[\left(I_{\max }+I_{\min }\right) / 2\right]^{-1}=10 \%$, with $I_{\max }$ $\left(I_{\min }\right)$ the intensity at the first peak (dip), is considerably underestimated.

\section{Results}

Our analysis clearly shows that there is an oscillation during the X-ray flare on AT Mic. The oscillation, which has a confidence level $>99.9 \%$, starts when the flare reaches its maximum and continues during the extended flare top. A damped sine curve with an oscillation period of $P=750 \mathrm{~s}$ and an exponential decay time of $\tau=2000 \mathrm{~s}$ fits the data. The initial relative amplitude is $\Delta I / I=0.15$.

In the following, we investigate the different types of magneto-acoustic loop oscillations. Comparing their various estimates for the flare loop length with two other, independent methods for the loop length, namely from radiative cooling times and from pressure balance, we find the most likely oscillation mode. We also derive the magnetic field strength.

\subsection{Magneto-acoustic waves}

Zaŭtsev \& Stepanov (1989) introduced a model, where a hard $\mathrm{X}$-ray oscillation is excited by a centrifugal force, which the evaporating plasma exerts on the flare loop, stretching the magnetic field lines upward and triggering Alfvén oscillations. In this scenario, the relative amplitude of the oscillation is determined by the additional energy from filling the magnetic flux tube with hot plasma

$\frac{\Delta I}{I} \approx \frac{4 \pi n k_{\mathrm{B}} T}{B^{2}}$

With $\Delta I / I=0.15, n=(4 \pm 3) \times 10^{10} \mathrm{~cm}^{-3}, T=(24 \pm 4) \mathrm{MK}$ and $k_{\mathrm{B}}$ the Boltzmann constant, the magnetic field then is $B=(105 \pm 50) \mathrm{G}$.

Roberts et al. (1984) showed that for a straight cylindrical geometry several types of magneto-acoustic wave modes in a magnetic loop are feasible, namely the slow (acoustic), the fast kink and the fast sausage modes. For a standing oscillation in a loop, the loop length $L$ is given by

$L=j c P / 2$,

with $P$ the oscillation period, $j$ the mode number and $c$ the appropriate wave speed. For slow modes, $c$ is the tube speed $c_{\mathrm{t}}$, with

$\frac{1}{c_{\mathrm{t}}^{2}}=\frac{1}{c_{\mathrm{s}}^{2}}+\frac{1}{c_{\mathrm{A}}^{2}}$,

whereas for the fast kink modes, $c$ is the kink speed $c_{\mathrm{k}}$, with

$c_{\mathrm{k}}^{2}=\frac{n c_{\mathrm{A}}^{2}+n_{\mathrm{e}} c_{\mathrm{Ae}}^{2}}{n+n_{\mathrm{e}}}$,

with the sound speed $c_{\mathrm{s}}=\sqrt{\gamma p / \rho}$ and the Alfvén speed $c_{\mathrm{A}}=B / \sqrt{4 \pi \rho} \cdot \gamma=5 / 3$ is the adiabatic constant for a monatomic gas/plasma, $p$ the gas pressure and $\rho$ the plasma density. For the fast sausage mode, $P=2 \pi a / c_{\mathrm{k}}$, where $a$ is the tube radius. We set $j=1$ for the fundamental mode, where the loop apex has maximum displacement, in agreement with the centrifugal model of Zaŭtsev \& Stepanov (1989).

The sound speed is $c_{\mathrm{s}}=\sqrt{2 \gamma k_{\mathrm{B}} T / m_{\mathrm{p}}}=1.66 \times 10^{4} \sqrt{T}=$ $(8.1 \pm 0.7) \times 10^{7} \mathrm{~cm} \mathrm{~s}^{-1}$. Using the centrifugal model and inserting Eq. (1) into the expression for the Alfvén speed, we obtain $c_{\mathrm{A}}=\left(k_{\mathrm{B}} T\right)^{1 / 2}\left[m_{\mathrm{p}}(\Delta I / I)\right]^{-1 / 2}=(1.1 \pm 0.1) \times 10^{8} \mathrm{~cm} \mathrm{~s}^{-1}$. We immediately see that, assuming the centrifugal model, the ratio of Alfvén to sound speed is determined by the relative oscillation amplitude and is $c_{\mathrm{A}} / c_{\mathrm{s}}=[2 \gamma(\Delta I / I)]^{-1 / 2} \approx 1.41$. Using this last relation, the tube speed then is $c_{\mathrm{t}} \approx 0.82 c_{\mathrm{s}}=$ $(6.6 \pm 0.6) \times 10^{7} \mathrm{~cm} \mathrm{~s}^{-1}$. To obtain the kink speed, we also need to know the Alfvén speed outside the loop, $c_{\text {Ae }}$. Assuming the same value for the magnetic field strength inside and outside the loop, $c_{\mathrm{Ae}}=c_{\mathrm{A}} \sqrt{n / n_{\mathrm{e}}}=(1.7 \pm 1.4) \times 10^{8} \mathrm{~cm} \mathrm{~s}^{-1}$. Note the large error because of the large density error. The kink speed then is $c_{\mathrm{k}}=c_{\mathrm{A}} \sqrt{2 n /\left(n+n_{\mathrm{e}}\right)}=(1.3 \pm 1.1) \times 10^{8} \mathrm{~cm} \mathrm{~s}^{-1}$. Thus, we have $\left(c_{\mathrm{t}}=0.82 c_{\mathrm{s}}\right)<c_{\mathrm{s}}<\left(c_{\mathrm{A}}=1.41 c_{\mathrm{s}}\right)<\left(c_{\mathrm{k}}=1.65 c_{\mathrm{s}}\right)<$ $\left(c_{\mathrm{Ae}}=2.05 c_{\mathrm{s}}\right)$.

Assuming a standing slow-mode oscillation, the loop length is $L_{\mathrm{sm}}=P c_{\mathrm{t}} / 2=(2.5 \pm 0.2) \times 10^{10} \mathrm{~cm}$. For a standing fast kink-mode oscillation, a loop length of $L_{\mathrm{fk}}=P c_{\mathrm{k}} / 2=$ $(5 \pm 4) \times 10^{10} \mathrm{~cm}$ is derived. And assuming a fast sausage mode oscillation, $a=P c_{\mathrm{k}} /(2 \pi)=(1.6 \pm 1.4) \times 10^{10} \mathrm{~cm}$ and $L_{\mathrm{fs}}=(8 \ldots 16) \times 10^{10} \mathrm{~cm}$, assuming an aspect ratio of $L / a=5 . . .10$.

\subsection{Loop length from radiative cooling times}

The loop length can also (and independently of any oscillation) be estimated from rising and cooling times obtained from the temporal shape of the flare, applying a flare heating/cooling model (see, e.g., Cargill et al. 1995). We follow the approach by Hawley et al. (1995) who investigated a flare on AD Leo observed in the extreme ultraviolet. The shape of this flare is very similar to our flare on AT Mic, but roughly 10 times larger, and shows a flat top, too.

The flare loop energy equation for the spatial average is given by

$\frac{3}{2} \dot{p}=Q-R$,

with $Q$ the volumetric flare heating rate, $R$ the optically thin cooling rate and $\dot{p}$ the time rate change of the loop pressure. During the rise phase, strong evaporative heating is dominant $(Q \gg R)$, while the decay phase is dominated by radiative cooling and strong condensation $(R \gg Q)$. At the loop top, there is an equilibrium $(R=Q)$. The loop length can be derived as

$L=\frac{1500}{\left(1-x_{\mathrm{d}}^{1.58}\right)^{4 / 7}} \cdot \tau_{\mathrm{d}}^{4 / 7} \cdot \tau_{\mathrm{r}}^{3 / 7} \cdot T^{1 / 2}$,

where $\tau_{\mathrm{r}}$ is the rise time, $\tau_{\mathrm{d}}$ the flare decay time (indicated in Fig. 2 with the vertical dotted lines), $T$ the apex flare temperature and $x_{\mathrm{d}}^{2}=c_{\mathrm{d}} / c_{\max }$, with $c_{\max }$ the peak count rate and $c_{\mathrm{d}}$ the count rate at the end of the flare. Inserting these values, the loop length is found to be $L \approx 2.5 \times 10^{10} \mathrm{~cm}$. 


\subsection{Pressure balance}

To maintain stable flare loops, the gas pressure of the evaporated plasma must be smaller than the magnetic pressure

$2 n k T \leq \frac{B^{2}}{8 \pi}$.

Knowing the flare density and temperature, we get a lower limit for the magnetic field strength $B>80 \pm 60 \mathrm{G}$. Again, there is a large error because of the large uncertainty for the density. Shibata \& Yokoyama (2002) assume pressure balance and give equations for $B$ and $L$ :

$B=50\left(\frac{E M}{10^{48} \mathrm{~cm}^{-3}}\right)^{-1 / 5}\left(\frac{n_{\mathrm{e}}}{10^{9} \mathrm{~cm}^{-3}}\right)^{3 / 10}\left(\frac{T}{10^{7} \mathrm{~K}}\right)^{17 / 10} \mathrm{G}$
$L=10^{9}\left(\frac{E M}{10^{48} \mathrm{~cm}^{-3}}\right)^{3 / 5}\left(\frac{n_{\mathrm{e}}}{10^{9} \mathrm{~cm}^{-3}}\right)^{-2 / 5}\left(\frac{T}{10^{7} \mathrm{~K}}\right)^{-8 / 5} \mathrm{~cm}$

Using these relations, we obtain a magnetic field strength of $B=75 \pm 40 \mathrm{G}$ and a loop length of $L=(2.8 \pm 1.7) \times 10^{10} \mathrm{~cm}$.

Combining the above results, we find that both pressure balance considerations as well as the centrifugal oscillation model are consistent. The derived loop lengths from pressure balance and radiative cooling times are consistent with each other and in agreement with the loop length derived assuming a slow-mode oscillation or a fast kink mode. The fast sausage mode is much less likely to be the cause of this oscillation, as its derived loop length is inconsistent with the other independent methods. We further discuss the plausibility of the different modes in the following section.

\section{Discussion and conclusions}

We have investigated an X-ray oscillation during a flare on AT Mic, observed by XMM-Newton. In order to locate the oscillation in time and frequency, we applied a continuous wavelet transform to the time series, and found that the oscillation, starting at flare peak and continuing during the flare's flat-top phase, with a period around $P=750 \mathrm{~s}$, has a confidence level of $>99.9 \%$ (Fig. 2). We then reconstructed the light curve from the CWT for three different frequency bands (Fig. 3). In particular, the light curve passed through a low-pass filter $(P>1200 \mathrm{~s}$, first panel), displays the overall shape of the light curve and picks out the long-lasting flares, whereas the light curve reconstructed from wavelet coefficients in the range around the oscillation period (500 s $<P<1200 \mathrm{~s}$, second panel), isolates the oscillation. The coherent oscillation during the large flare can be fitted with a damped sine curve with a period of $P=750 \mathrm{~s}$ and an exponential decay time of $\tau=2000 \mathrm{~s}$ (Fig. 4). The initial, relative peak-to-peak amplitude of the oscillation is found to be $\Delta I / I=15 \%$. Decomposing a light curve in such a way is a powerful tool for isolating an oscillation from a flare, as well as disentangling the flare and the oscillation from the high-frequency noise. Because the effect of noise can thus be greatly reduced, this method provides us with a more accurate value for the oscillation amplitude than a value potentially obtained from a binned or smoothed light curve, which is always underestimated, especially if the bins are large.
Table 1. Comparison of values derived for the indicated models.

\begin{tabular}{lll}
\hline \hline Model & Loop length & Magnetic field \\
\hline Slow mode $^{a}$ & $(2.5 \pm 0.2) \times 10^{10} \mathrm{~cm}$ & \\
Fast kink mode $^{a}$ & $(5 \pm 4) \times 10^{10} \mathrm{~cm}$ & \\
Fast sausage mode $^{a}$ & $(8 \ldots 16) \times 10^{10} \mathrm{~cm}$ & \\
Centrifugal force model $^{b}$ & & $(105 \pm 50) \mathrm{G}$ \\
\hline Pressure balance $^{c}$ & $(2.8 \pm 1.7) \times 10^{10} \mathrm{~cm}$ & $(75 \pm 40) \mathrm{G}$ \\
Radiative cooling $^{d}$ & $2.5 \times 10^{10} \mathrm{~cm}$ & \\
${ }^{a}$ Roberts et al. (1984). & \\
${ }^{b}$ Zaĭtsev \& Stepanov (1989). \\
${ }^{c}$ Shibata \& Yokoyama (2002). \\
${ }^{d}$ Hawley et al. (1995).
\end{tabular}

Interpreting this oscillation as a standing magneto-acoustic wave in the flare loop, we infer that it is a longitudinal slowmode wave, oscillating at the fundamental frequency. This mode has an anti-node (maximum disturbance) at the loop apex and is capable of causing the largest global flux variations. Using the relations derived by Roberts et al. (1984), we find a flare loop length of $L=(2.5 \pm 0.2) \times 10^{10} \mathrm{~cm}$. This value is consistent with estimating the loop length from radiative cooling times of the flare (Hawley et al. 1995) as well as from pressure balance considerations (Shibata \& Yokoyama 2002). Table 1 provides a comparison of these results. The derived loop length of a fast kink wave, which has a large error due to a large error in density, is also consistent with the two independent methods of radiative cooling times and pressure balance. This mode, however, is basically incompressible (Nakariakov et al. 2004), and it would be hard to imagine how it could cause intensity perturbations in a spatially unresolved light curve. We discount fast sausage-mode waves, since they would require either much longer loops (several times the stellar radius) for the observed period, which is inconsistent with the loop length derived from the radiative cooling as well as from pressure balance models, or a loop aspect ratio $(L / a)$ close to 1 , in which case the cylindrical geometry assumption breaks down. In summary, we conclude that the observed oscillation is most likely a standing longitudinal slow-mode wave.

To estimate the magnetic field strength, we apply the centrifugal force model of Zautsev \& Stepanov (1989), where the magnetic loop is stretched by a centrifugal force which is caused by the upwardly evaporating plasma, stretching the loop beyond equilibrium and thus exciting MHD waves. The flux tube starts to oscillate up and down (i.e. in the plane of the loop). Consequently, the particle density inside the loop also oscillates, causing an oscillation in the thermal radiation (soft X-rays and extreme ultra-violet). Non-thermal radiation, originating from gyro-synchrotron (radio) and bremsstrahlung (hard X-rays and optical), caused by fast electrons trapped within the loop and moving back and forth from one end of the loop to the other, also oscillates: the speed of the trapped electrons, and hence the non-thermal radiation, is modulated by the plasma density. This picture is consistent with a slow-mode (acoustic) wave. The magnetic field strength thus obtained is $B=(105 \pm 50) \mathrm{G}$. The large error results from the large uncertainty in the particle density. This value for the magnetic 
field strength is consistent with pressure balance considerations (Shibata \& Yokoyama 2002), where $B=(75 \pm 40) \mathrm{G}$.

The observed rapid damping of the oscillation (a damping time comparable to the oscillation period) is in agreement with other magneto-acoustic wave observations (e.g., Ofman \& Wang 2002; Verwichte et al. 2004). So far, various models have been put forward to explain the fast damping. In the case of slow-mode waves, Ofman \& Wang (2002) numerically find that thermal conduction is the dominant dissipation mechanism in loops with $T \geq 6 \mathrm{MK}$.

An alternative to the interpretation of a standing magnetoacoustic wave causing the flare oscillation is that repeated and rapid flaring is occurring. This might also explain the flat-top character of the time profile of this flare, which differs from the usual shape of solar flares, which shows a rapid rise from beginning to peak, followed by a slow decay (see, e.g., Švestka 1989). However, repeated flaring should be random, rather than have a periodic and exponentially-decaying nature, and would have difficulties explaining the observation of what appears to be a damped oscillation.

The derived length of the flare loop is about the size of the stellar radius and is comparable to loop lengths of large solar flares. In particular, Švestka (1994) investigated solar X-ray observations which showed periods close to $20 \mathrm{~min}$. They associated them with spatially resolved large-scale coronal loops with loop lengths of $(2-3) \times 10^{10} \mathrm{~cm}$, and interpreted the oscillations as slow-mode MHD waves. Terekhov et al. (2002) observed an X-ray oscillation with $P=143$ s during a solar flare, and derived a loop length of $(1-3) \times 10^{10} \mathrm{~cm}$. The centrifugal force model, originally derived for non-thermal X-rays (Zaĭtsev \& Stepanov 1989), has also been applied to millimetre-wave emission (Stepanov et al. 1992), thermal X-rays (Terekhov et al. 2002), and, in the stellar case, to optical emission (Mullan et al. 1992; Mathioudakis et al. 2003).

This is the first time that an oscillation has been observed in X-rays in a stellar flare, and has been used to derive a flare loop length and a local magnetic field strength. Comparable loop dimensions and magnetic field strengths indicate the similar natures of AT Mic's corona and that of our Sun. Because of the smaller radius of these M-type stars $\left(r_{\star} \approx 0.5 r_{\odot}\right)$, the loop length is in fact of the order of the stellar radius, whereas the magnetic field strength is around the upper limit for solar coronal values. This is consistent with this class of low mass stars (dMe-type) being very X-ray active.

Acknowledgements. We would like to thank the referee D. J. Mullan for calling to our attention a missed reference and a couple of inconsistencies in the text. We would also like to thank V. M. Nakariakov, B. Roberts, M. Mathioudakis, N. Schartel, G. Peres, A. J. J. Raassen, R. Erdélyi, E. Verwichte, and J. L. Culhane for various helpful discussions and comments. We acknowledge financial support from the UK Particle Physics and Astronomy Research Council (PPARC).

\section{Appendix A: CWT error analysis}

The continuous wavelet transform of the signal $f$ is given by convolving the signal with a wavelet function $\Psi$

$W=\Psi * f$ or in components:

$W_{j n}=\sum_{m} \Psi_{j n m}^{*} \cdot f_{m}$,

with $W_{j n}=W\left(t_{n}, s_{j}\right), \Psi_{j n m}=\Psi\left(\frac{(n-m) \Delta t}{s_{j}}\right)=\sqrt{\frac{\Delta t}{s_{j}}} \Psi_{0}\left(\frac{(n-m) \Delta t}{s_{j}}\right)$, $f_{m}=f\left(t_{m}\right)$, the ${ }^{*}$ denoting the complex conjugate and the scale $s_{j}=s_{0} 2^{j \delta j}$. In our analysis, we set $s_{0}=1$ and $\delta j=0.25$ for the Morlet wavelet. Significance levels can be derived as

$$
\begin{aligned}
\sigma_{j n}^{\mathrm{Re}} & =\sqrt{\sum_{m} \mathcal{R} e\left(\Psi_{j n m}\right)^{2} \cdot \sigma_{m}^{2}} \\
\sigma_{j n}^{\mathrm{Im}} & =\sqrt{\sum_{m} \mathcal{I} m\left(\Psi_{j n m}\right)^{2} \cdot \sigma_{m}^{2}} \\
\chi^{2} & =\left(\frac{\mathcal{R} e\left(W_{j n}\right)}{\sigma_{j n}^{\mathrm{Re}}}\right)^{2}+\left(\frac{\mathcal{I} m\left(W_{j n}\right)}{\sigma_{j n}^{\mathrm{Im}}}\right)^{2} \equiv \chi_{2}^{2}
\end{aligned}
$$

for $\sigma_{m}^{2}$ the variance of $f_{m}$, and $\chi^{2}$ the $\chi^{2}$-distribution of $W_{j n}$. The signal can be reconstructed by using

$f_{n}=c \cdot \sum_{j} \frac{\operatorname{Re}\left\{W_{j n}\right\}}{\sqrt{s_{j}}}$,

with $c$ the normalisation constant (see, e.g., Torrence \& Compo 1998). If a filter $F$ is applied to the wavelet coefficients, $\widetilde{W}_{j n}=W_{j n} \cdot F_{j n}$, the filter coefficients being 0 or 1 , the filtered signal is then given by

$f_{n}=c \cdot \sum_{j} \frac{\mathcal{R} e\left\{\widetilde{W}_{j n}\right\}}{\sqrt{s_{j}}}$.

Inserting Eqs. (A.2) into (A.7) and rearranging the sums, we obtain

$f_{n}=\sum_{m} A_{m n} f_{m}$,

with

$A_{m n}=c \cdot \sum_{j} \frac{\operatorname{Re}\left\{\Psi_{j n m}\right\} \cdot F_{j n}}{\sqrt{s_{j}}}$.

Thus, the signal can be filtered and reconstructed using a linear transform, which is determined by the wavelet function and the filter. The variance of the reconstructed light curve is then given by

$\sigma_{n}^{2}=\sum_{m} A_{m n}^{2} \sigma_{m}^{2}$

The signal can be decomposed without loss, if the sum of the different filters used is 1 for all filter components.

\section{References}

Andrews, A. D. 1990, A\&A, 239, 235

Aschwanden, M. J., Fletcher, L., Schrijver, C. J., \& Alexander, D. 1999, ApJ, 520, 880

Cargill, P. J., Mariska, J. T., \& Antiochos, S. K. 1995, ApJ, 439, 1034

Gliese, W., \& Jahreiss, H. 1991, Preliminary Version of the Third Catalogue of Nearby Stars, Astron. Rechen-Institut, Heidelberg 
Handy, B. N., Acton, L. W., Kankelborg, C. C., et al. 1999, Sol. Phys., 187,229

Hawley, S. L., Fisher, G. H., Simon, T., et al. 1995, ApJ, 453, 464

Jansen, F., Lumb, D., Altieri, B., et al. 2001, A\&A, 365, L1

Lim, J., Nelson, G. J., \& Vaughan, A. E. 1987, Proc. ASA, 7, 2

Mathioudakis, M., Seiradakis, J. H., Williams, D. R., et al. 2003, A\&A, 403, 1101

Mitra-Kraev, U., Harra, L. K., Güdel, M., et al. 2005, A\&A, 431, 679

Mullan, D. J., \& Johnson, M. 1995, ApJ, 444, 350

Mullan, D. J., Herr, R. B., \& Bhattacharyya, S. 1992, ApJ, 391, 265

Nakariakov, V. M., \& Ofman, L. 2001, A\&A, 372, L53

Nakariakov, V. M., Tsiklauri, D., Kelly, A., Arber, T. D., \& Aschwanden, M. J. 2004, A\&A, 414, L25

Ofman, L., \& Wang, T. 2002, ApJ, 580, L85

Perryman, M. A. C., Lindegren, L., Kovalevsky, J., et al. 1997, A\&A, 323, L49
Raassen, A. J. J., Mewe, R., Audard, M., \& Güdel, M. 2003, A\&A, 411, 509

Roberts, B. 2000, Sol. Phys., 193, 139

Roberts, B., Edwin, P. M., \& Benz, A. O. 1984, ApJ, 279, 857

Rodonò, M. 1974, A\&A, 32, 337

Shibata, K., \& Yokoyama, T. 2002, ApJ, 577, 422

Stepanov, A. V., Urpo, S., \& Zaŭtsev, V. 1992, Sol. Phys., 140, 139

Strüder, L., Briel, U., Dennerl, K., et al. 2001, A\&A, 365, 18

Terekhov, O. V., Shevchenko, A. V., Kuz'min, A. G., et al. 2002, Astron. Lett., 28, 397

Torrence, C., \& Compo, G. P. 1998, Bull. Amer. Meteor. Soc., 79, 61 Verwichte, E., Nakariakov, V. M., Ofman, L., \& DeLuca, E. E. 2004, Sol. Phys., 223, 77

Švestka, Z. 1989, Sol. Phys., 121, 399

Švestka, Z. 1994, Sol. Phys., 152, 505

Zătsev, V. V., \& Stepanov, A. V. 1989, Sov. Astron. Lett., 15, 66 